\title{
Current and future research efforts in oligometastatic non-small cell lung cancer-a systematic review
}

\author{
Mariana Brandão ${ }^{1}$, Valérie Durieux ${ }^{2}$, Thierry Berghmans ${ }^{1}$ \\ ${ }^{1}$ Clinic of Thoracic Oncology, Institut Jules Bordet, Université Libre de Bruxelles, Brussels, Belgium; ${ }^{2}$ Bibliothèque des Sciences de la Santé, \\ Université Libre de Bruxelles, Brussels, Belgium \\ Contributions: (I) Conception and design: All authors; (II) Administrative support: V Durieux; (III) Provision of study materials or patients: V Durieux, \\ M Brandão; (IV) Collection and assembly of data: V Durieux, M Brandão; (V) Data analysis and interpretation: All authors; (VI) Manuscript writing: \\ All authors; (VII) Final approval of manuscript: All authors. \\ Correspondence to: Thierry Berghmans, MD, PhD. Clinic of Thoracic Oncology, Institut Jules Bordet, Rue Héger-Bordet, 1, B-1000 Brussels, \\ Belgium. Email: thierry.berghmans@bordet.be.
}

\begin{abstract}
Background: Major progresses in the systemic treatment of non-small cell lung cancer (NSCLC) were obtained during the last decade, including the use of immunotherapy and tyrosine kinase inhibitors (TKIs), with impressive results in terms of response and survival rates. Moreover, novel imaging and radiotherapy techniques have allowed the development of stereotactic body radiotherapy (SBRT), with high rates of local disease control and minimal toxicity. These factors propelled the use of combined systemic and local treatment strategies in patients with a low burden of metastases-the oligometastatic disease (OMD).

Methods: We systematically review the evidence from prospective randomized and non-randomized trials on local ablative therapy for OMD NSCLC published until June 2020. In addition, we present a review of the ongoing and/or recruiting trials in the field.

Results: We included 16 articles, reporting on 14 prospective clinical trials, starting from the pilot trial conducted in the early 2000's to the recent randomized trials that have showed benefits in survival. We found 24 ongoing trials, which combine multiple local ablative regimens with new systemic therapies, such as new generation TKIs and immunotherapy.

Discussion: Despite these vast current and ongoing prospective research efforts, there are several issues that impair the generalizability of their findings. These include the heterogeneous definition of OMD, trial design, staging, patient selection, tumor mutational status and treatments used, which may limit their applicability in the clinical practice.
\end{abstract}

Keywords: Oligometastatic; non-small cell lung cancer (NSCLC); stereotactic radiotherapy; surgery; chemotherapy; immunotherapy

Submitted Aug 20, 2020. Accepted for publication May 17, 2021.

doi: $10.21037 /$ tlcr-20-964

View this article at: http://dx.doi.org/10.21037/tlcr-20-964

\section{Introduction}

Major progresses in the treatment of non-small cell lung cancer (NSCLC) were obtained during the last decade. Introduction of adjuvant chemotherapy after surgery or adjuvant immunotherapy after concomitant radio- chemotherapy led to improved survival rates in local and locally advanced NSCLC. In stage IV disease, the discovery of targetable oncogenic driver mutations/ translocations and immune checkpoints (PD-1/PD-L1/ CTLA-4) led to development of specific drugs (tyrosine

\footnotetext{
^ ORCID: Mariana Brandão, 0000-0002-1653-3544; Valérie Durieux, 0000-0002-8975-6562; Thierry Berghmans, 0000-0001-7447-2519.
} 
kinase or immune checkpoints inhibitors) with impressive data both in terms of response and survival rates. However, stage IV is a continuum status from single to multiple and diffuse metastatic sites. In 1995, Hellman et al. proposed the concept of oligometastatic disease (OMD) (1). This new concept corresponds to an intermediate state between locoregional and classical metastatic diseases with distinct prognoses, and potential benefit from local ablative therapy. Outside the agreement about the meaning of "oligo" as few/limited number of metastases, no consensual OMD definition is currently accepted despite proposals from scientific societies as the European Society for Medical Oncology (ESMO) (2). The European Organisation for Research and Treatment of Cancer (EORTC) Lung Cancer Group designed an initiative aiming to propose a consensual definition of synchronous OMD (sOMD) to be used in clinical trials (3). The OMD concept covers different clinical situations that can be summarized in four settings: sOMD, occurring at the time of initial NSCLC diagnosis; oligorecurrence (rOMD), developing after optimal local control of a localized tumor; oligoprogression (pOMD), corresponding to a progression of disease in a limited number of metastatic sites; and oligopersistant disease (peOMD) after/on systemic therapy.

A number of retrospective series focusing on specific metastatic sites as adrenal, lung or brain were summarized in systematic reviews (4-6) showing potential interest of primary and metastatic sites radical treatment in increasing local control and prolonging survival. Obviously, not all patients with OMD are benefiting from this aggressive approach as patients with controlled primary tumor, with $\mathrm{N}$ stage $0-1$ and metachronous (vs. synchronous) OMD deserved better survival (7). This therapeutic approach was further tested in nonrandomized studies with a first pilot study showing the feasibility of surgery both on the primary and the single metastatic sites after induction chemotherapy, with 57\% of complete resections and 11 months of median overall survival (OS) (8).

The aim of this article is to systematically review the published evidence from prospective randomized and non-randomized trials on local ablative therapy for OMD NSCLC. In addition, a review of the ongoing and/or recruiting trials in the field will be presented. We present the following article in accordance with the PRISMA reporting checklist (available at http://dx.doi.org/10.21037/ tlcr-20-964).

\section{Methods}

The literature search was performed in June 2020 using the Ovid Medline system. This research was performed by a scientific librarian (VD), experienced in searching for medical and scientific publications, and by physicians, experts in the treatment of thoracic neoplasms and trained in evidence-based medicine. Ovid Medline database was searched using the OvidSP interface. The "Population, Intervention, Comparison, Outcome" (PICO) questions model for clinical questions was used to identify the concepts included in the questions. The corresponding search criteria of "P" and "I" were translated into MeSH terms, free-text keywords and name of substances or interventions (Table S1). Results were limited to prospective, phase II or phase III studies. Citations were exported from Medline into reference manager databases (EndNote) to allow the removal of duplicates and to facilitate the selection process performed by reviewers. All articles retrieved by the librarian selected for their eligibility by two authors based on the title and abstract and the final selection was performed by reading the full publication and its inclusion was consensually decided. This search was supplemented by screening the references of the selected articles and other literature known by the experts. One author (MB) retrieved information concerning study design (including staging assessments, tumor molecular status, and number of allowed metastases), sample size, treatment arms and efficacy outcomes in terms of progression-free survival (PFS) and OS.

In order to review the ongoing trials in the OMD field, we performed a search in the clinicaltrials.gov registry from inception to July 30, 2020. We used the following search criteria: (I) search terms: "oligometastatic" OR "oligometastases" OR "SBRT"; (II) condition or disease: NSCLC; (III) recruitment status: recruiting, not yet recruiting, active, not recruiting, enrolling by invitation; (IV) age groups: adult and older adult; phase: 2, 3, 4, and not applicable. In order to be eligible, trials had to be prospective therapeutic interventional phase II-IV studies and to specifically include patients with OMD NSCLC (as defined by each trial), either as the main population of the trial or as a pre-defined subgroup for outcome analysis. Studies limited to the treatment of brain metastases and/ or studies that have already published their final results were excluded. We retrieved information regarding study name, country, design, sample size, mandatory staging procedures, molecular status of patients' tumors, 

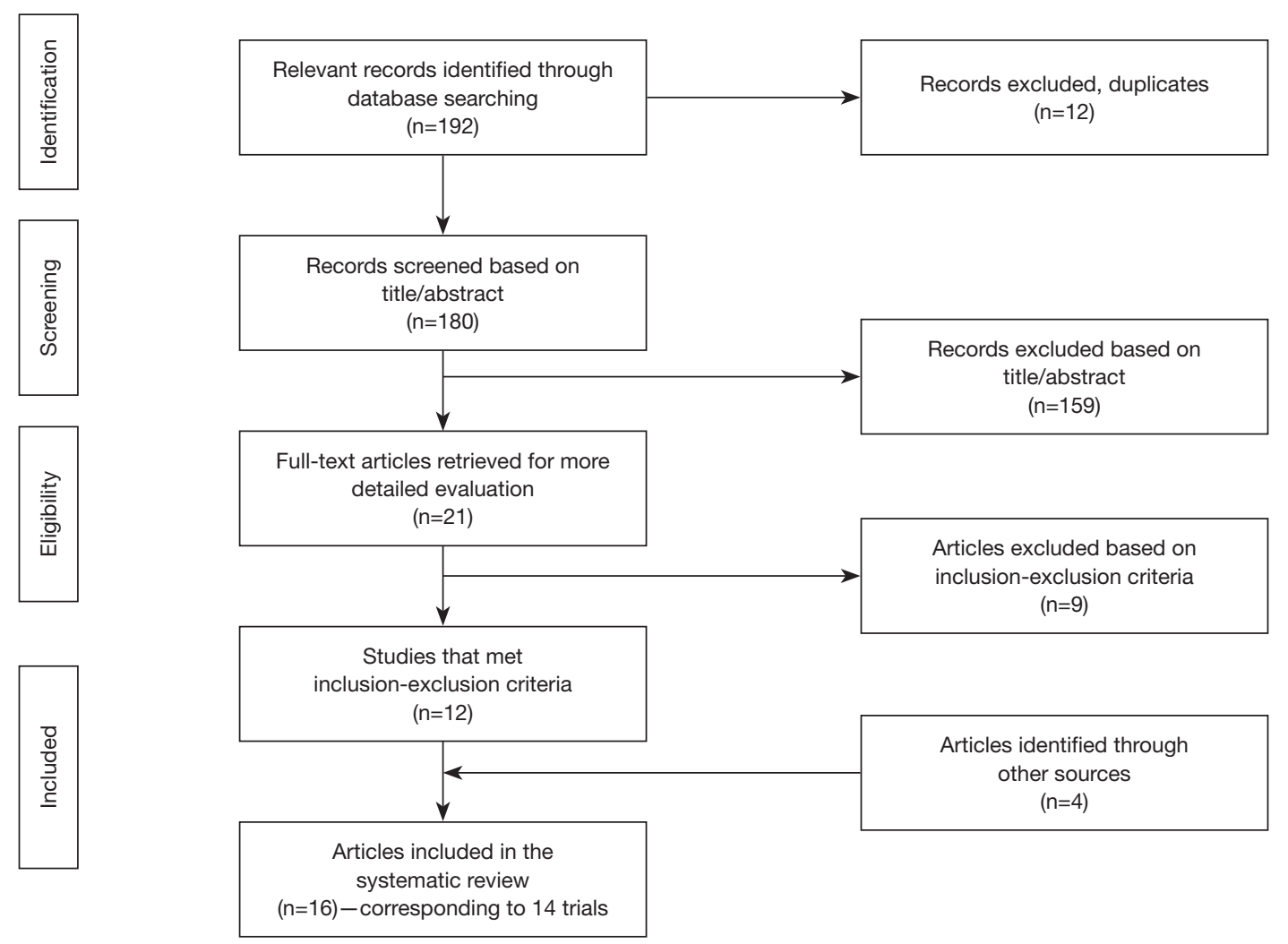

Figure 1 Published trials flowchart.

maximum number of metastases allowed, treatment arm(s) and endpoints.

\section{Current prospective evidence}

The systematic search of literature yielded 180 citations and, after screening for title/abstract, 21 references were submitted to full-manuscript review. Of these, 12 articles met the inclusion/exclusion criteria; moreover, four additional articles were retrieved from other sources. Therefore, 16 articles, reporting on 14 prospective clinical trials, were included in this review (Figure 1). Definition of "oligometastatic" state ranged from a single metastasis to 6 metastatic lesions in total (Table 1). However, in all trials, over $50 \%$ of patients included had $\leq 2$ metastatic sites of disease.

\section{$s O M D$}

The first prospective trial in patients with OMD was published by Downey et al. (8) in the early 2000's, including
23 patients with stage IV NSCLC with a synchronous single metastasis. Patients were proposed to receive three cycles of chemotherapy, followed by surgery to all sites of disease and two additional cycles of chemotherapy. Patients were young (median age of 55 years), half were women, and 14/23 patients had a solitary brain metastasis. Only 14/23 patients were submitted to a lung resection, as the other 9 patients had progressive disease while on chemotherapy. The median OS was 11 months, and there were two patients who were still alive 5 years after diagnosis.

Subsequent single-arm trials explored the effect of surgery and/or radiotherapy, either as concomitant chemoradiotherapy, stereotactic body radiotherapy (SBRT) or stereotactic radiosurgery (SRS), in sOMD (9-16). The number of patients included ranged from 20 to 198 and only three of these trials mandated both the performance of ${ }^{18} \mathrm{~F}$-FDG PET and brain imaging as baseline staging $(10,13,16)$, as recommended by the EORTC consensus (3). In most of these trials, the tumor's molecular status was unknown (10,12-16), but on the Arrieta trial, $41 \%$ of patients had an EGFR-mutated tumor and 3\% had an 
Table 1 Published/presented phase II-IV clinical trials on the treatment of oligometastatic NSCLC

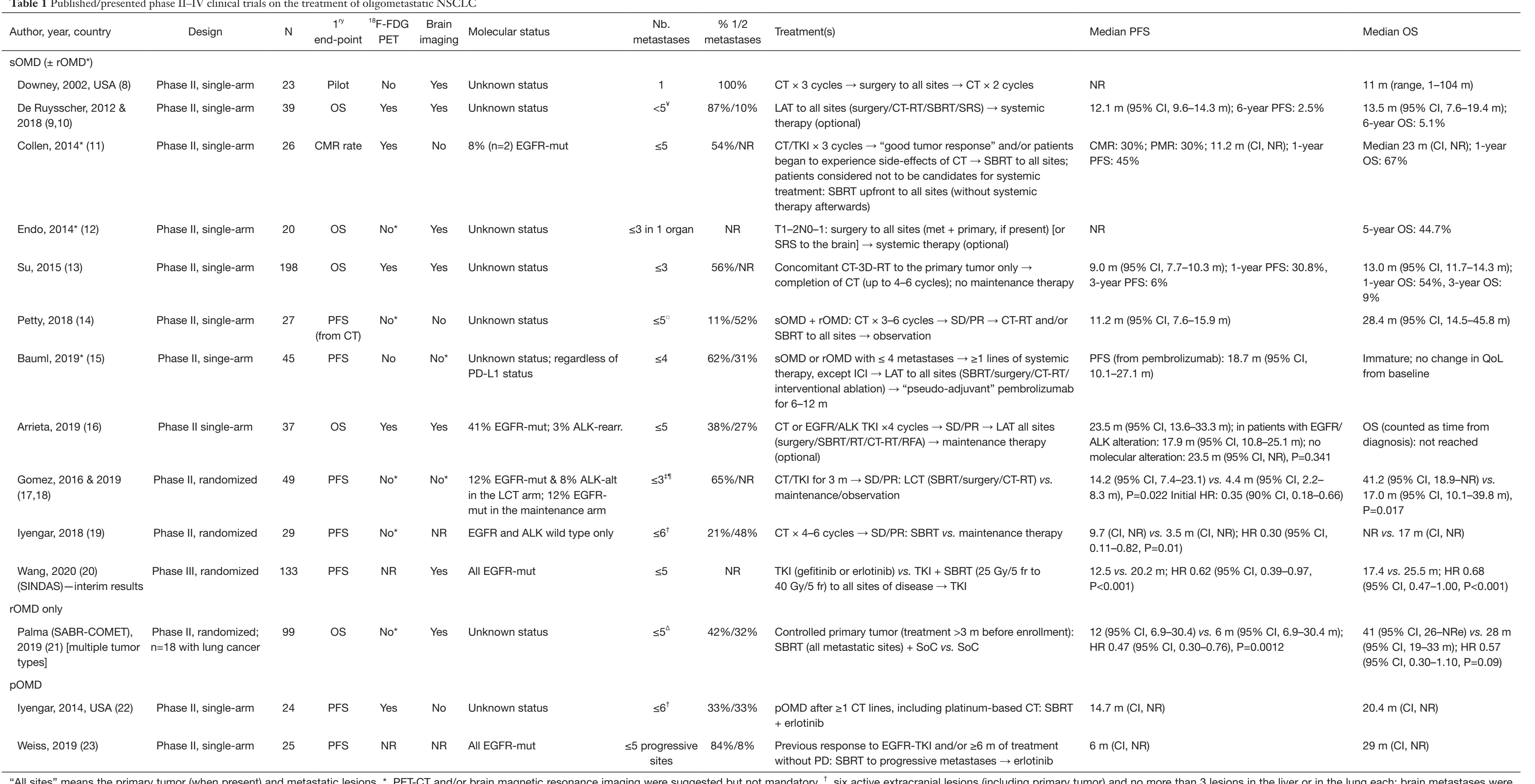

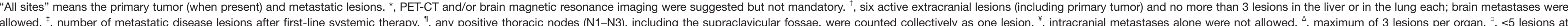

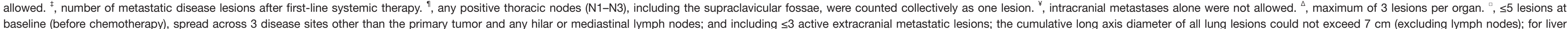

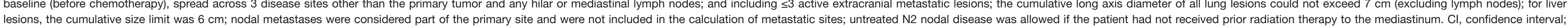

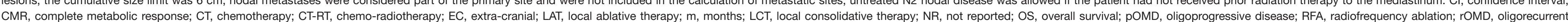
disease; RT, radiotherapy; SBRT, stereotactic body radiotherapy; SoC, standard of care treatment; SRS, stereotactic radiosurgery; sOMD, synchronous oligometastatic disease. 
ALK-rearranged tumor (16).

In the De Ruysscher trial, patients with $\leq 5$ metastases were submitted to upfront local ablative therapy (surgery, chemo-radiotherapy, SBRT and/or SRS) to all sites of disease, with optional systemic therapy afterwards (10). In the Su trial, patients received concomitant chemoradiotherapy only to the primary tumor, followed by the completion of chemotherapy (up to 4-6 cycles), but without systemic maintenance therapy (13). The median PFS and OS were similar between the two trials (12.1 and 9.0 months; and 13.5 and 13.0 months, respectively). On the other hand, Endo et al. only included patients with T1-2N0 -1 tumors and $\leq 3$ metastases in a single organ, who were submitted to surgery to all sites of disease (or SRS to the brain) followed by optional systemic therapy (12). These tight selection criteria may explain the reported 5-year OS of $44.7 \%$.

Other trials opted for a design in which patients started with systemic therapy and, in the absence of progression, were submitted to local therapy $(11,14-16)$. After a variable course of chemotherapy or tyrosine kinase inhibitor (TKI), patients in the Collen trial received SBRT to all sites of disease (11). Alternatively, patients who were considered not to be candidates for systemic treatment because of medically unfit condition and/or low tumor burden or patients' refusal, could underwent SBRT as upfront treatment (without systemic therapy afterwards). This was the only trial having as primary endpoint the achievement of a complete metabolic response, as defined by ${ }^{18} \mathrm{~F}$-FDG PET, which was of $30 \%$. Petty enrolled patients that received 3-6 cycles of chemotherapy, without progression, and submitted them to chemo-radiotherapy and/or SBRT to all sites of disease, followed by observation; in this trial, both PFS and OS were measured from date of chemotherapy initiation, instead of date of enrolment in the trial (14). In the Arrieta trial, patients without progression after four cycles of chemotherapy or TKI received local ablative therapy to all sites, followed by optional maintenance therapy (16). In a more recent trial, by Bauml et al., patients under systemic therapy [except an immune checkpoint inhibitor (ICI)] underwent local ablative therapy, followed by a "pseudo-adjuvant" course of pembrolizumab for 6-12 months (15). The heterogeneity in patient population and study designs may explain why median PFS ranged from 11.2 to 23.5 months and OS from 23 months to "not reached" in these studies.

In the sOMD setting, there are two randomized phase II trials that have been published as a full manuscript (17-19) and the phase III randomized SINDAS trial that recently presented its interim results at the ASCO Congress 2020 (20). Gomez included patients regardless of their tumor's molecular status, while Iyengar only enrolled patients without oncogenic driver alterations (in EGFR or ALK). In both trials, patients received systemic therapy for 3-4 months. In the absence of progression, they were randomized to local treatment (SBRT in Iyengar et al.; local consolidative treatment, consisting of SBRT, surgery and/ or chemo-radiotherapy in Gomez et al.) vs. maintenance therapy/observation. Both trials reported a significant improved in PFS, with hazard ratios between $0.30[95 \%$ confidence interval (CI), 0.11-0.82] and 0.35 (90\% CI, 0.18-0.66). Moreover, Gomez showed an impressive increase in median OS from 17.0 to 41.2 months.

On the other hand, only patients with an EGFR-mutated tumor were included in the SINDAS trial and randomized to standard EGFR-TKI therapy vs. EGFR-TKI plus SBRT to all sites of disease, followed by maintenance EGFR-TKI (20). Among the first 133 patients included, the median PFS significantly improved from 12.5 to 20.2 months with the addition of SBRT and the median OS increased from 17.4 to 25.5 months. The publication of the final results is now expected.

\section{Other OMD settings}

The phase II randomized SABR-COMET enrolled 99 patients with a controlled primary tumor and oligorecurrent disease, of whom 18 had lung cancer (21). Patients were randomized to SABR to all metastatic sites plus standardof-care $v s$. standard-of-care. Overall, there was a significant prolongation of PFS and a numerical increase in OS, which was not significant.

In addition, two phase II single-arm trials explored the use of SBRT to oligoprogressive sites of disease $(22,23)$. The first study enrolled patients regardless of their tumor's mutational status, who underwent SBRT plus concurrent erlotinib and had a median OS of 20.4 months (22). More recently, Weiss et al. performed a prospective single-arm trial based on the current recommendations of management of patients who previously benefited from EGFR-TKI therapy and that develop oligoprogressive disease (23): patients underwent SBRT to oligoprogressive sites and continued treatment with a TKI (erlotinib). Their median PFS was only 6 months, but the median OS was 29 months. This trial also tested the prognostic role of Veristrat, a serum proteomic signature, which was predictive of differential benefit from erlotinib in the second-line 


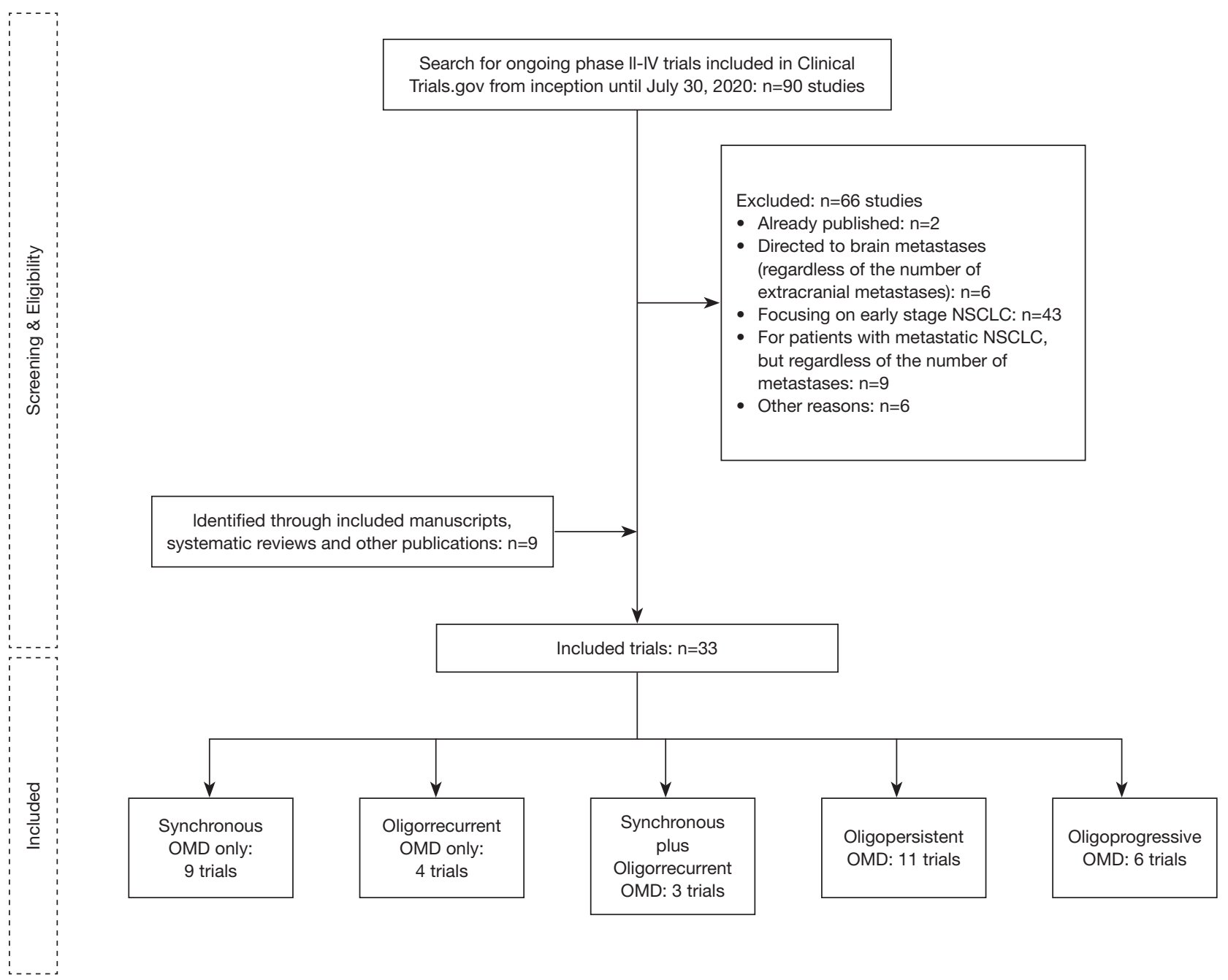

Figure 2 Ongoing trials selection flow chart. NSCLC, non-small cell lung cancer; OMD, oligometastatic disease.

treatment of unselected NSCLC patients (24). Yet, among patients included in the Weiss study, this signature could not predict PFS (23).

\section{Future research efforts}

We retrieved 90 studies in clinicaltrials.gov, of which 24 trials were included (Figure 2). Through the included manuscripts, systematic reviews and other publications we identified 9 additional ongoing trials. There were 33 trials included, aiming to enroll a total of 4,255 patients, and with a median number of 100 patients per trial (https://cdn. amegroups.cn/static/public/tlcr-20-964-1.pdf) (25-27). Almost half of these studies are being carried in the synchronous and/or oligorecurrent setting and most of them are randomized (70\%).

\section{Local treatment strategies}

Given the progresses observed in the last decade in radiotherapy techniques, all ongoing trials in OMD NSCLC use radiotherapy as one of the possible or the only locally ablative treatment allowed. Most trials recommend SBRT, either alone, or in conjunction with conventionally fractioned or hypofractioned radiotherapy or concurrent chemo-radiotherapy to the primary tumor. When reported, regimens of SBRT differ widely across trials, but in most cases, they are not described or are left at the discretion of the treating investigator. One of the trials allows the prescription of SBRT with photons or protons 
(NCT02314364). Another one uses stereotactic ablation brachytherapy (NCT04486287). Other ablative techniques include surgery and radiofrequency ablation. The phase III randomized OMEGA trial (NCT03827577) mandates surgical resection of the primary lung tumor (if still in place), but in all other trials, ablation of the primary tumor may also be performed using radiotherapy or radiotherapy is the sole treatment modality permitted.

\section{Systemic therapies}

Immunotherapy has been recently introduced in the treatment of NSCLC, which explains why, so far, only one published trial in OMD enrolled patients who received ICI (15). Given the important survival benefits brought by immunotherapy in the treatment of NSCLC, it is surprising that the number of ongoing trials specifically mentioning a combination of local ablative therapy with immunomodulatory drugs is still small (30\%). As expected, some of them use already approved ICI for NSCLC, such as durvalumab [CHESS trial (NCT03965468); NCT04255836], pembrolizumab [NRG LU002 (NCT03137771)], the combination of nivolumab with ipilimumab [LONESTAR trial (NCT03391869)] the use of standard-of-care immunotherapy, but without specifying which type [SARON (NCT02417662), SUPPRESSNSCLC (NCT04405401)]. Other studies are using drugs that have not yet been approved, such as darleukin [ImmunoSABR (NCT03705403)]; TQB2450 [antiPD-L1 agent; (NCT04306926)]; recombinant human granulocyte macrophage stimulating factor [rhGMCSF, in CRAGMOLC (NCT03489616)]; and sintilimab (NCT04486287) and SHR-1210 (NCT03557411), which are both anti-PD-1 drugs.

Among these trials, the CHESS trial (NCT03965468) is an especially interesting study, as it will combine and alternate the use of immunotherapy (durvalumab) and chemotherapy (carboplatin and paclitaxel) with radiotherapy (SBRT and conventional radiotherapy) and surgery for $\mathrm{SOMD}$. It has the objective of potentiate the immunomodulatory effects of radiotherapy and will also have an associated translational research program, which will better clarify the biology of OMD.

Trials specifically including patients with oncogenic driver alterations mandate the use of EGFR/ALK-TKI and two of them specifically combine osimertinib with local ablative treatment (NCT03667820 and NCT03410043). Most trials for "all comers" also recommend the use of
TKIs as systemic therapy in case the patient's tumor has an oncogenic driver alteration, following current clinical practice (28). However, most trials do not specify which type of systemic therapy the patient should receive and recommend that the physician should choose the standardof-care systemic therapy available.

\section{Phase II/III and III randomized trials}

There is an impressive number of 11 ongoing phase II/ III and phase III randomized trials for OMD, of which 7 only include NSCLC patients. In the sOMD setting, the large SARON trial (NCT02417662) is including 340 patients without oncogenic driver alterations, randomized to chemotherapy with/without radical radiotherapy to the primary tumor and all metastatic sites. Importantly, patients are stratified by hospital, histology, nodal stage ( $\mathrm{N} 0 / 1$ vs. N2/3), number of oligometastatic sites ( $1 v s .2 / 3)$, brain metastases (present $v s$. absent) and its primary outcome is OS.

The OITROLOC trial (NCT02076477) is prescribing ablative radiotherapy to all patients and its aim is to understand the best timing for radiotherapy in OMD: either upfront or after two cycles of chemotherapy. The already discussed Sindas trial (NCT02893332) is testing the addition of consolidative SBRT to EGFR-TKI in patients with EGFR-mutated tumors.

Then, for patients with a previously treated primary tumor and that have now an $\mathrm{OOMD}$, both the CORE (NCT02759783) and the SABR-COMET 10 (NCT03721341) studies are evaluating if the addition of SBRT to standardof-care therapy improves survival outcomes among patients with different tumor types. The main difference between them is that the CORE has a limit of 3 metastatic sites, while the SABR-COMET 10 has the same design as the already published SABR-COMET trial (21), but it includes patients with a higher burden of disease (4 to 10 metastatic sites).

The OMEGA (NCT03827577) and STEREO-OS (NCT03143322) studies are including patients both with sOMD and with rOMD. The OMEGA trial has a similar design to CORE and SABR-COMET 10, but also allows the use of surgery for the treatment of the primary tumor. Interestingly, the STEREO-OS is only including patients with $\leq 3$ bone metastases, either from breast, prostate or NSCLC, who are randomized to systemic treatment with/ without SBRT, plus local ablative treatment of the primary tumor, if still in place.

The NRG LU002 trial (NCT03137771) is targeting 
a different patient population, i.e., those who have OMD after induction systemic therapy, regardless of the number of metastases at baseline. This includes patients with "genuine" OMD (i.e., oligometastatic since diagnosis) or "induced" OMD (i.e., patients with a previous history of polymetastatic disease) (29). This large trial is testing whether the addition of SBRT and/or chemo-radiotherapy to standard maintenance therapy may prolong PFS and OS. The LONESTAR study (NCT03391869) is including patients with both polymetastatic and OMD NSCLC, receiving nivolumab/ipilimumab and without evidence of progression after 12 weeks of therapy. The trial is assessing if the addition of local consolidative treatment to the immunotherapy combination improves OS, both in the overall population and in the OMD group.

For patients with oligoprogressive disease, there are two phase II/III randomized trials currently ongoing. The PROMISE-004 study (NCT03808662) includes patients with breast or NSCLC with $\leq 5$ progressing metastases in one single extra-cranial organ, who are randomized to receive upfront SBRT to all oligoprogressive sites $v s$. standard-of-care therapy only. The HALT trial (NCT03256981) is only enrolling patients with oncogenic driver alterations, that benefited from TKI therapy and that have now a progression of disease in $\leq 3$ sites. Patients are randomized in a 2:1 fashion to continue with the same TKI therapy with/without SBRT. The trial is therefore testing if the current recommendation of performing SBRT to oligoprogressive sites maintaining the same TKI improves PFS or not (28).

\section{Limitations of current and ongoing studies}

Despite these vast current and ongoing prospective research efforts to understand which therapies may be beneficial to NSCLC patients with OMD, there are several issues that impair the generalizability of these studies' findings. Here we discuss some of them.

\section{Definition of OMD}

The first and very important caveat is in the definition of OMD itself. In published prospective studies, the number of metastatic sites ranged from 1 to 6 , but most of the patients included had $\leq 2$ sites of metastatic disease (54-100\%). Moreover, the way of "counting" the number of sites is also heterogeneous. Some trials included the primary tumor $(19,22)$, while all the others did not. One study collectively counted any positive thoracic nodes (N1N3) as one lesion (17), while other excluded these thoracic nodes from the counting (14) and Endo et al. only allowed patients with N0-1 status (12). Recently, the EORTC panEuropean consensus proposed the definition of sOMD as $\leq 5$ metastases in $\leq 3$ organs, in which mediastinal lymph nodes are not counted as a metastatic site, but are rather considered regional disease (3). Still, the consensus' participants agreed that prospective data collection and clinical trials are needed to improve this current definition. Moreover, they also proposed that future clinical trials should consider stratification according to thoracic lymph node status-which is being followed by the SARON trial (NCT02417662).

However, there is still a wide variation in the definition of OMD in ongoing studies, as $27 \%$ of included trials define it as $\leq 3$ metastatic lesions, $48 \%$ as $\leq 5$ metastatic sites, $12 \%$ use other cut-offs and $12 \%$ do not define OMD in their inclusion/exclusion criteria. Moreover, there is still variability in the way of counting N1-N3 lymph nodes: in the SARON and NCT04255836 trials they are not counted as a metastatic lesion, while they are collectively counted as one lesion in NCT01725165, and each progressing lymph node is counted as a separate lesion in the SUPPRESSNSCLC trial (NCT04405401).

Regarding lesions' dimensions, there are trials defining a maximum size per lesion, such as in the SABR-COMET 10 (NCT03721341) study, in which extra-cranial lesions have to be $\leq 5 \mathrm{~cm}$ of diameter and brain metastase $\leq 3 \mathrm{~cm}$ or with a total volume $\leq 30 \mathrm{cc}$. These requirements are usually related to the potential increased toxicity of performing radiotherapy in larger lesions, but other trials prefer to leave this feasibility assessment to the physician/multidisciplinary tumor board, in a case-by-case basis.

So far, all current definitions of OMD are based on imaging, i.e., on the number, size and location of metastases. There are efforts towards the development of biomarkers to define OMD (30), but none has been prospectively validated so far. Therefore, as imaging modalities evolve, data obtained from older trials and/or studies, such as the one defining synchronous NSCLC into risk groups (7) may be no longer applicable.

\section{Trial design}

Most of the published studies so far are non-randomized and, therefore, do not provide conclusive evidence regarding the benefit of adding local ablative therapy to 
systemic therapy in patients with OMD. The randomized trials by Gomez and Iyengar $(17,19)$ suggested that patients receiving systemic therapy for 3-4 months, with stable disease or partial response, benefit from the addition of local consolidative therapy and they were even closed earlier due to these positive findings. Nonetheless, the number of patients included was still small and their primary endpoint was PFS. The SABR-COMET trial (21) also demonstrated a benefit of using SBRT in patients with oligorecurrent disease, but it only included 18 patients with NSCLC. The SINDAS trial showed promising interim results (20), but the publication of the full results is awaited. Fortunately, there is a large number of ongoing randomized studies including NSCLC patients with OMD and given that most of them use radiotherapy as the local ablative therapy (instead of surgery) and include patients in different disease settings, they will probably be able to accrue the necessary number of subjects.

Nonetheless, most of these trials use PFS as their main endpoint $(67 \%)$ and only $24 \%$ of trials have OS as a primary endpoint. This is not ideal, given that all these studies are unblinded and that PFS is a surrogate endpoint, with an imperfect correlation with OS (31). Still, one may argue that in such a symptomatic disease like NSCLC, the use of PFS is a valid endpoint, as it may correlate with an improvement in the quality-of-life. Interestingly, 13 of the ongoing studies (56\%) include quality-of-life as a secondary endpoint, which is an important evolution, given that, so far, only Bauml has prospectively reported this endpoint in the treatment of OMD (15).

Moreover, there were trials that measured survival not since enrollment in the study (as usually recommended) but from diagnosis of NSCLC (16) or from date of chemotherapy or TKI initiation (14) (NCT03916913), which may have / will artificially increase survival outcomes reported in these studies.

In terms of disease setting, both Gomez and Iyengar $(17,19)$ enrolled patients with metastatic NSCLC after a course of systemic therapy, regardless of the number of metastases at diagnosis. Therefore, they have included both patients with "genuine" and "induced" OMD, but its respective proportions were not provided, which may hamper the applicability of their findings in clinical practice. On the other hand, they also triggered the hypothesis that the tumor's sensitivity to systemic therapy may be more important in the definition of OMD than the number of baseline metastases, as it may give a hint to the tumor's biological behavior.
Currently, there are three ongoing trials also defining OMD based on the number of metastases after induction systemic therapy [NCT01725165; CRAGMOLC (NCT03489616), NRG LU002 (NCT03137771)], but all other trials define OMD based on the number of baseline metastases.

\section{Work-up/staging}

The EORTC Consensus mandates the use of ${ }^{18} \mathrm{~F}$-FDG PET and brain imaging as baseline staging for the definition of OMD (3). Nonetheless, as previously seen, only three of the published studies had ${ }^{18}$ F-FDG PET and brain imaging as obligatory procedures $(10,13,16)$. The absence of these mandatory screening exams may have led to occult imbalances between treatment arms in the Gomez and Iyengar randomized trials regarding the patients' burden of disease and, potentially, influenced disease outcomes $(17,19)$. Most ongoing trials $(64 \%)$ do not specifically mention the use of ${ }^{18} \mathrm{~F}$-FDG PET or brain imaging in their inclusion/exclusion criteria, therefore it is not possible to know whether it is a requirement or not. Still, randomized trials such as SARON (NCT02417662), OMEGA (NCT03827577), STEREO-OS (NCT03143322) and ImmunoSABR (NCT03705403) mandate its use, which will improve the strength and generalization of their findings.

Moreover, the use of ${ }^{18} \mathrm{~F}$-FDG PET accurately identifies mediastinal lymph node involvement, for which it has a high sensitivity and specificity (32). This is an important aspect, as some trials count mediastinal lymph nodes as a site of disease, define the treatment to the primary tumor according to it and/or use it as a stratification factor. With the good sensitivity of ${ }^{18} \mathrm{~F}$-FDG PET in assessing mediastinal invasion, histological confirmation is rarely reported. The EORTC consensus proposes that histological confirmation of at least one metastasis is performed unless the multidisciplinary tumor board decides that the risk outweighs the benefit.

\section{Patient selection}

Like in most clinical trials in general, patients included in the published studies were younger and had a better performance status (usually WHO or ECOG 0-1) compared to the general population of patients with metastatic NSCLC. De Ruysscher also pointed out that patients included in their study had less comorbidities ( $46 \%$ 
with no comorbidities; $51 \%$ with one comorbidity) (10). Likewise, in the ongoing trials, patients are usually required to have a performance status of $0-1$ at enrollment and no serious comorbidities.

Published studies enrolled patients with brain metastases, which were usually included in the metastatic sites count. Nonetheless, both studies from Iyengar $(19,22)$ allowed the inclusion of patients with treated/stable brain metastases, but these were not counted for the definition of OMD. Currently, most ongoing studies do not allow the inclusion of patients with intracranial metastases alone, as these are already treated with local ablative treatments (surgery, SRS and/or stereotactic ablative radiotherapy). Moreover, some trials allow the inclusion of patients with intracranial metastases, but consider that they are treated by standardof-care radiotherapy and do not take them into account in the number of metastatic lesions [e.g., NCT02314364, PROMISE-004 (NCT03808662), HALT (NCT03256981)], which is in conflict with the EORTC definition and will impair comparison of results with other trials.

A retrospective study suggested that patients with squamous OMD treated with radical intention (systemic therapy plus local ablative therapy) had a worse OS compared to patients with adenocarcinoma (33). In all published trials, adenocarcinoma was the most frequent histology, but in the Arrieta study, almost $95 \%$ patients presented with nonsquamous histology and $44 \%$ had a tumor with an oncogenic driver alteration, which may explain the very long median PFS (23.5 months) reported (16). On the other hand, there was a frank imbalance between arms on the proportion of patients with squamous NSCLC in the Gomez and Iyengar randomized studies $(17 \%$ and $20 \%$ in the maintenance treatment arm vs. $4 \%$ and $7 \%$ in the local consolidative therapy arm, respectively), which may also have influenced outcomes $(17,19)$. Both the SARON and ImmunoSABR randomized trials include histological subtype as a stratification factor $(25,26)$, but this is not the case of the SABR-COMET-10 study, as it includes multiple tumor types (27) nor of the OMEGA trial (NCT03827577).

Another important factor to consider is the inclusion of patients with tumors with oncogenic driver alterations in each trial. Again, in the Gomez study, there was an imbalance regarding the proportion of patients with an oncogenic driver alteration in the local consolidative therapy (20\%) compared to the maintenance arm (8\%) (17). On the contrary, Iyengar only included patients without EGFR or ALK-altered tumors (19) and the SINDAS and Weiss studies were directed only to patients with EGFR-mutated tumors $(20,23)$. However, in most of the other published trials, the tumor's molecular status was unknown, which limits the generalization of their findings $(8,10,12-15,21,22)$.

Ongoing studies are following a trend of separating patients with oncogene-addicted tumors from those with no actionable molecular aberration. Nonetheless, there are still trials directed to "all comers", but some of them include the presence of oncogene-addicted tumors as a stratification/dynamic balancing factor (e.g., OMEGA trial, NCT03827577).

The only trial reporting so far the proportion of patients with PD-L1 positive tumors was the one by Bauml et al., testing "pseudo-adjuvant" pembrolizumab after local ablative treatment. They found a numerical improvement in 24-month PFS among patients with PD-L1-positive compared to those with PD-L1-negative tumors $(69.3 \%$ vs. $38.1 \%$, respectively; non-significant) (15), which is line with other trials testing pembrolizumab in metastatic NSCLC. The OMEGA trial (NCT03827577) includes PD-L1 status as a dynamic balancing factor, but the other trials do not mention it in their clinicaltrials.gov description.

\section{Treatment selection}

Unlike the classic randomized studies testing systemic drugs in metastatic NSCLC, in which treatment arms are very well-defined, the published and ongoing randomized trials in OMD are usually more flexible regarding the therapy(ies) that can be used in each arm.

Many trials do not specify which type of systemic therapy the patient should receive and recommend that the physician should choose the standard-of-care systemic therapy available. This is a pragmatic approach, which reflects current clinical practice and allows the incorporation of newly approved drugs in the trial. However, new distant metastases are usually the most common sites of disease progression in patients included in OMD trials, highlighting the importance of having effective systemic therapy to control micrometastatic disease that is not ablated by local treatments (9). Therefore, trials' outcomes are influenced not only by the local ablative strategies, but also by the type of systemic therapy used. This limits the generalization of their survival and toxicity findings to other countries/regions in which some types of systemic therapy may not be available (e.g., immunotherapy combinations, novel TKIs, etc.). 
Furthermore, in case of oligoprogressive disease, it is not clear if systemic therapy should be maintained or switched to a new line. Most current trials in oligoprogressive disease maintain the same systemic therapy in the treatment arm that receives local ablative therapy, as a way to control resistant clones, while keeping the sensible clones under the same therapy. Nonetheless, there are two single-arm studies that switch treatment to new immunotherapy drugs (NCT04486287 and NCT03557411).

Furthermore, there is a large heterogeneity in terms of the local ablative strategies used. Earlier trials employed surgery as the main ablative approach $(8,12)$, while subsequent trials moved to a "mixed" strategy of combining surgery with radiotherapy, according to physician's choice $(10,15,17)$ or to the exclusive use of radiotherapy, i.e., chemo-radiotherapy and/or SBRT (11,13,14,16,19-23). Radiotherapy dosing schedules varied widely across trials and even within trials, according to the site and size of metastases (e.g., intracranial vs. extracranial). Ongoing trials also employ many different radiotherapy regimens, thus it is difficult to assess which are the most effective strategies. Moreover, the optimal timing of combining radiotherapy with systemic therapy (e.g., immunotherapy, TKIs) is also unknown, with some trials using sequential schemes [e.g., SARON trial (NCT02417662), NRG LU002 trial (NCT03137771)], while others deliver them concurrently [e.g., CHESS trial (NCT03965468)]. The OITROLC trial (NCT02076477) is trying to answer this question in sOMD, comparing upfront chemo-radiotherapy, followed by chemotherapy $v s$. the inverted strategy.

Other ablative techniques, such as radiofrequency ablation, are being explored, but only in two ongoing trials [NCT02805530; OMEGA (NCT03827577)].

Given the difficulty of testing all the different combinations of systemic therapy and local ablative regimens, real-life prospective registries, such as the OligoCare cohort (NCT03818503), may help understanding the benefits and risk of each of these strategies and combinations.

\section{Conclusions}

During the last decade, there was a growing interest in combining local ablative and systemic therapies in oligometastatic NSCLC. Despite encouraging results in terms of PFS, and eventually OS, coming from randomized and non-randomized phase II studies, the question of considering OMD as a separate therapeutic entity from classic stage IV NSCLC remains, as the available data are lacking generalizability mainly due to some scientific limitations and introduction of immunotherapy as a firstline standard of care systemic therapy. Further, OMD is a heterogeneous setting needing standardization in terms of definition, with clear specification of the excluded sites that cannot be amenable to a local therapy (pleural effusion, carcinomatous meningitis, etc.) and maximum number of metastatic sites/lesions, extensive work-up, and harmonization of systemic therapies, as proposed in the EORTC LCG initiative (3). In the future, specific trials including tumors with oncogenic driver mutations should be preferred to the "all-comers" design, considering the major therapeutic impact of very active drugs in an evolving domain with recently tested compounds as for example in RET translocation or KRAS G12C mutation.

Major efforts are currently developed in clinical research and large phase III trials are ongoing. It is of importance that the scientific community supports those trials by including eligible patients so that many questions regarding therapeutic strategy in OMD NSCLC can be resolved in the next years.

\section{Acknowledgments}

Funding: None.

\section{Footnote}

Provenance and Peer Review: This article was commissioned by the editorial office, Translational Lung Cancer Research for the series "Oligometastatic NSCLC: definition and treatment opportunities". The article has undergone external peer review.

Reporting Checklist: The authors have completed the PRISMA reporting checklist. Available at http://dx.doi. org/10.21037/tlcr-20-964

Conflicts of Interest: All authors have completed the ICMJE uniform disclosure form (available at http://dx.doi. org/10.21037/tlcr-20-964). The series "Oligometastatic NSCLC: definition and treatment opportunities" was commissioned by the editorial office without any funding or sponsorship. TB serves as the unpaid Guest Editor of the series. MB received speaker honorarium and travel grant from Roche/GNE. TB received consulting fees from Inhatarget, payment from Bayer and participation fees for advisory board from Roche, Bayer and Janssen. The authors 
have no other conflicts of interest to declare.

Ethical Statement: The authors are accountable for all aspects of the work in ensuring that questions related to the accuracy or integrity of any part of the work are appropriately investigated and resolved.

Open Access Statement: This is an Open Access article distributed in accordance with the Creative Commons Attribution-NonCommercial-NoDerivs 4.0 International License (CC BY-NC-ND 4.0), which permits the noncommercial replication and distribution of the article with the strict proviso that no changes or edits are made and the original work is properly cited (including links to both the formal publication through the relevant DOI and the license). See: https://creativecommons.org/licenses/by-nc-nd/4.0/.

\section{References}

1. Hellman S, Weichselbaum RR. Oligometastases. J Clin Oncol 1995;13:8-10.

2. Novello S, Barlesi F, Califano R, et al. Metastatic nonsmall-cell lung cancer: ESMO Clinical Practice Guidelines for diagnosis, treatment and follow-up. Ann Oncol 2016;27:v1-27.

3. Dingemans AC, Hendriks LEL, Berghmans T, et al. Definition of synchronous oligometastatic non-small cell lung cancer-a consensus report. J Thorac Oncol 2019;14:2109-19.

4. Pfannschmidt J, Dienemann H. Surgical treatment of oligometastatic non-small cell lung cancer. Lung Cancer 2010;69:251-8.

5. Siva S, MacManus M, Ball D. Stereotactic radiotherapy for pulmonary oligometastases: a systematic review. J Thorac Oncol 2010;5:1091-9.

6. Collaud S, Stahel R, Inci I, et al. Survival of patients treated surgically for synchronous single-organ metastatic NSCLC and advanced pathologic TN stage. Lung Cancer 2012;78:234-8.

7. Ashworth A, Rodrigues G, Boldt G, et al. Is there an oligometastatic state in non-small cell lung cancer? A systematic review of the literature. Lung Cancer 2013;82:197-203.

8. Downey RJ, Ng KK, Kris MG, et al. A phase II trial of chemotherapy and surgery for non-small cell lung cancer patients with a synchronous solitary metastasis. Lung Cancer 2002;38:193-7.

9. De Ruysscher D, Wanders R, Hendriks LE, et al.
Progression-free survival and overall survival beyond 5 years of NSCLC patients with synchronous oligometastases treated in a prospective phase II trial (NCT 01282450). J Thorac Oncol 2018;13:1958-61.

10. De Ruysscher D, Wanders R, van Baardwijk A, et al. Radical treatment of non-small-cell lung cancer patients with synchronous oligometastases: long-term results of a prospective phase II trial (Nct01282450). J Thorac Oncol 2012;7:1547-55.

11. Collen C, Christian N, Schallier D, et al. Phase II study of stereotactic body radiotherapy to primary tumor and metastatic locations in oligometastatic nonsmall-cell lung cancer patients. Ann Oncol 2014;25:1954-9.

12. Endo C, Hasumi T, Matsumura Y, et al. A prospective study of surgical procedures for patients with oligometastatic non-small cell lung cancer. Ann Thorac Surg 2014;98:258-64.

13. Su S, Li T, Lu B, et al. Three-dimensional radiation therapy to the primary tumor with concurrent chemotherapy in patients with stage IV non-small cell lung cancer: results of a multicenter phase 2 study from PPRA-RTOG, China. Int J Radiat Oncol Biol Phys 2015;93:769-77.

14. Petty WJ, Urbanic JJ, Ahmed T, et al. Long-term outcomes of a phase 2 trial of chemotherapy with consolidative radiation therapy for oligometastatic nonsmall cell lung cancer. Int J Radiat Oncol Biol Phys 2018;102:527-35.

15. Bauml JM, Mick R, Ciunci C, et al. Pembrolizumab after completion of locally ablative therapy for oligometastatic non-small cell lung cancer: a phase 2 trial. JAMA Oncol 2019;5:1283-90.

16. Arrieta O, Barrón F, Maldonado F, et al. Radical consolidative treatment provides a clinical benefit and long-term survival in patients with synchronous oligometastatic non-small cell lung cancer: a phase II study. Lung Cancer 2019;130:67-75.

17. Gomez DR, Blumenschein GR Jr, Lee JJ, et al. Local consolidative therapy versus maintenance therapy or observation for patients with oligometastatic non-smallcell lung cancer without progression after first-line systemic therapy: a multicentre, randomised, controlled, phase 2 study. Lancet Oncol 2016;17:1672-82.

18. Gomez DR, Tang C, Zhang J, et al. Local consolidative therapy vs. maintenance therapy or observation for patients with oligometastatic non-small-cell lung cancer: long-term results of a multi-institutional, phase II, randomized study. J Clin Oncol 2019;37:1558-65. 
19. Iyengar P, Wardak Z, Gerber DE, et al. Consolidative radiotherapy for limited metastatic non-small-cell lung cancer: a phase 2 randomized clinical trial. JAMA Oncol 2018;4:e173501.

20. Wang Y, Wang X, Guan Y, et al. Stereotactic radiosurgery combined with anlotinib for limited brain metastases with perilesional edema in non-small cell lung cancer: Rvision-001 study protocol. Thorac Cancer 2020;11:1361-4.

21. Palma DA, Olson R, Harrow S, et al. Stereotactic ablative radiotherapy versus standard of care palliative treatment in patients with oligometastatic cancers (SABRCOMET): a randomised, phase 2, open-label trial. Lancet 2019;393:2051-8.

22. Iyengar P, Kavanagh BD, Wardak Z, et al. Phase II trial of stereotactic body radiation therapy combined with erlotinib for patients with limited but progressive metastatic non-small-cell lung cancer. J Clin Oncol 2014;32:3824-30.

23. Weiss J, Kavanagh B, Deal A, et al. Phase II study of stereotactic radiosurgery for the treatment of patients with oligoprogression on erlotinib. Cancer Treat Res Commun 2019;19:100126.

24. Gregorc V, Novello S, Lazzari C, et al. Predictive value of a proteomic signature in patients with non-small-cell lung cancer treated with second-line erlotinib or chemotherapy (PROSE): a biomarker-stratified, randomised phase 3 trial. Lancet Oncol 2014;15:713-21.

25. Lieverse RIY, Van Limbergen EJ, Oberije CJG, et al. Stereotactic ablative body radiotherapy (SABR) combined with immunotherapy (L19-IL2) versus standard of care in stage IV NSCLC patients, ImmunoSABR: a multicentre, randomised controlled open-label phase II trial. BMC Cancer 2020;20:557.

26. Conibear J, Chia B, Ngai Y, et al. Study protocol for the SARON trial: a multicentre, randomised controlled phase

Cite this article as: Brandão $M$, Durieux V, Berghmans T. Current and future research efforts in oligometastatic non-small cell lung cancer-a systematic review. Transl Lung Cancer Res 2021;10(7):3473-3485. doi: 10.21037/tlcr-20-964
III trial comparing the addition of stereotactic ablative radiotherapy and radical radiotherapy with standard chemotherapy alone for oligometastatic non-small cell lung cancer. BMJ Open 2018;8:e020690. Erratum in: BMJ Open 2019;9:e020690corr1.

27. Palma DA, Olson R, Harrow S, et al. Stereotactic ablative radiotherapy for the comprehensive treatment of 4-10 oligometastatic tumors (SABR-COMET-10): study protocol for a randomized phase III trial. BMC Cancer 2019;19:816.

28. Planchard D, Popat S, Kerr K, et al. Metastatic nonsmall cell lung cancer: ESMO Clinical Practice Guidelines for diagnosis, treatment and follow-up. Ann Oncol 2018;29:iv192-237. Erratum in: Ann Oncol 2019;30:863-70.

29. Guckenberger M, Lievens Y, Bouma AB, et al. Characterisation and classification of oligometastatic disease: a European Society for Radiotherapy and Oncology and European Organisation for Research and Treatment of Cancer consensus recommendation. Lancet Oncol 2020;21:e18-28.

30. Lussier YA, Khodarev NN, Regan K, et al. Oligo- and polymetastatic progression in lung metastasis(es) patients is associated with specific microRNAs. PLoS One 2012;7:e50141. Erratum in: PLoS One 2013.

31. Berghmans T, Pasleau F, Paesmans M, et al. Surrogate markers predicting overall survival for lung cancer: ELCWP recommendations. Eur Respir J 2012;39:9-28.

32. Volpi S, Ali JM, Tasker A, et al. The role of positron emission tomography in the diagnosis, staging and response assessment of non-small cell lung cancer. Ann Transl Med 2018;6:95.

33. Kwint M, Walraven I, Burgers S, et al. Outcome of radical local treatment of non-small cell lung cancer patients with synchronous oligometastases. Lung Cancer 2017;112:134-9. 
Supplementary

Table S1 List of MeSH terms and free-text keywords used to search Ovid Medline database

\begin{tabular}{|c|c|}
\hline PICO criteria & Searched MeSH terms, free-text keywords and phrases \\
\hline I = oligometastatic & $\begin{array}{l}\text { oligometastas\#s or oligometastatic or (isolated adj3 metastas\#s) or (isolated adj3 metastatic) or } \\
\text { (limited adj3 metastas\#s) or (limited adj3 metastatic) or single organ metastatic or single organ } \\
\text { metastas\#s or solitary metastas\#s or solitary metastatic or oligoprogression or oligoprogressive). } \\
\text { ti,ab }\end{array}$ \\
\hline
\end{tabular}

Database: Epub Ahead of Print, In-Process \& Other Non-Indexed Citations, Ovid Medline ${ }^{\circledR}$ Daily and Ovid Medline ${ }^{\circledR}$ 1946-present. term/, $\mathrm{MeSH}$ term (with all the possible subheading combinations); exp, exploted MeSH term, meaning that this MeSH term and all the MeSH terms found below in the hierarchy are taken into consideration; .ti,ab, terms are searched in the title and the abstract; ADJn, terms are $\mathrm{n}$ words apart; *, stands for zero or more characters; \#, stands for one single character. 